\section{6 AUREOMYCIN, A NEW ANTIBIOTIC}

A

VERY important series of sixteen papers on aureomycin has been published in the Annals of the New York Academly of Sciences, vol. 51, Art. 2, pp. 175-342 (Nove fiter 30,1948 ). These papers were the result of gererence on aureomycin held by the Division of $\mathrm{f}$ cology of the Section of Biology, the New Yoh Academy of Sciences, on July 21, 1948. Thpy fopresent the work of forty-three investigators, sifle of whom are on the scientific staff of the Lederly Laboratories Division, American Cyanamid Company, Pearl River, N.Y. The remainder are well known and independent biological and medical research workers in official laboratories, university medical schools and hospitals, all of whom were supplied with aureomycin by the Lederle Laboratories.

In the introductory paper, B. M. Duggar, of the Lederle Laboratories, traces the history of the discovery of the new antibiotic, and pays tribute to his late colleague, Dr. Y. SubbaRow, who directed the work until his death. Aureomycin, which has been obtained as a highly purified, crystalline, faintly golden-yellow substance, is a metabolic product of Streptomyces aureofaciens, a hitherto undescribed species of the Actinomycetes. Hence its name, and not, as a London evening newspaper has recently stated, "because it can be given by the mouth". It has clearly been made in considerable quantitiesfermentation in tanks is mentioned-but few details are given of the methods used for the production and isolation of aureomycin, and chemists will look in vain for any information as to its chemical nature, except what can be gleaned from the fact that a hydrochloride and a borate are referred to. To avoid confusion, mention should be made of the fact that the trade mark 'Duomycin' has been given to aureomycin.

In vitro bacteriological studies of aureomycin, earried out in the Division of Penicillin Control and Immunology, Food and Drug Administration, Washington, in Johns Hopkins University School of Medicine and in Boston City Hospital and Harvard University Medical School, have established that aureomycin is effective against both Gram-negative and Gram-positive bacteria. Higher concentrations of the substance are required to produce bacterio. stasis than are necessary with penicillin, being close to those required of streptomycin. Bacteria do not readily become resistant to it in vitro. It seems to be rather unstable in aqueous solution even at room temperature, though the dry solid material appears to be quite stable. An exhaustive study of the pharmacology of aureomycin, carried out in the Lederle Laboratories and at Johns Hopkins, established that it has a low toxicity and almost no side reactions, particularly in solutions of $p \mathbf{H} \mathbf{8 \cdot 5}$.

P. A. Little, in the Lederle Laboratories, found aureomycin effective in experimental infections in mice of Eberthella typhosa, Erysipelothrix rhusiopathice, the causative agent of swine erysipelas, and also in chickens infected with the causative agents of fowl cholera and fowl typhoid. On the other hand, workers at Johns Hopkins failed to protect mice with aureomycin administered orally in experimental infections with Pneumococcus type I or $K$. pneumonice A., although it did protect against Streptococcus hemolyticus beta, and Dr. Henry Welch and his colleagues at the Food and Drug Administration, Washington, conclude that "no outstanding usefulness for this antibiotic has been demonstrated by our animal investigations against the bacteria studied. The greatest promise for the drug lies in the treatment of certain viral and rickettsial diseases."

In spite of the not altogether favourable laboratory findings, clinical trials of aureomycin in human patients have given very promising results. About a hundred patients with a variety of bacterial in. fections have been treated with aureomycin by mouth at the Boston City Hospital. The results obtained in cases of gonorrhoea, while indicating that this treatment was effective in many cases, were inferior to those obtained with adequate amounts of penicillin. Four patients with pneumococcal pneumonia were successfully treated with aureomycin by mouth. In a number of urinary tract infections the results were favourable, and may be comparable with or possibly superior to those obtained from the use of streptomycin in similar cases. In contrast to streptomycin, there was no evidence of the development of bacterial resistance to aureomycin in vivo. Infections with Proteus vulgaris and Pseudomonas aeruginosa were not benefited by aureomycin. The results in the cases of typhoid fever and of severe Salmonella infections were equivocal. Workers in the George Washington University Medical Departments also failed to obtain any clinical or bacteriological response in four patients with typhoid fever. The results obtained by workers at Johns Hop.kins in four cases of primary atypical pneumonia were very similar to those of the Boston workers. Eight cases of chronic urinary tract infection, which had not responded to penicillin, streptomycin or sulphonamide therapy, cleared promptly with aureo. mycin. A. E. Braley and M. Sanders, of the Department of Ophthalmology, College of Physicians and Surgeons, Columbia University, New York, report favourably on results with aureomycin borate used locally and aureomycin hydrochloride used intramuscularly in two hundred patients with a wide range of ocular infections, and found the antibiotic to be effective against some of the Gram-positive cocci and several Gram-negative bacilli. It was also found to be an effective therapeutic agent in the virus in fections, inclusion canjunctivitis and herpes simplex of the cornea.

Indeed, even more striking than the antibacterial activities of aureomycin are its effects on viruses and rickettsiæ. S. C. Wong and H. R. Cox, of the Lederle Laboratories, have shown that although it has no apparent in vitro activity, it has marked therapeutic activity against the viruses of the psittacosislymphogranuloma group and rickettsiæ of the spotted fever, typhus fever, scrub typhus fever and $Q$ fever groups in embryonated hens' eggs, mice and guinea pigs. On the other hand, it failed to show any therapeutic activity against a number of other viral infections, notably the $B$ strain of influenza, canine distemper and the $M E F-1$ strain of poliomyelitis. Investigators in the rickettsial research laboratory of the University of Texas report favourable results in the treatment of guinea pigs infected with spotted fever and epidemic typhus. Clinical trials of aureomycin in human patients suffering from four different types of viral or rickettsial infections have given very promising results. A group of workers at Johns Hopkins and other investigators in the George Washington University Medical Departments obtained quite dramatic cures in a total of twenty.one cases of Rocky Mountain spotted fever treated with oral aureomycin. Workers in the Harlem Hospital 
and Columbia University, New York, treated a total of thirty-five cases of lymphogranuloma venereum, usually by intramuscular injection, and conclude from the excellent results they obtained that aureomycin is the treatment of choice in this virus infection. They also found that the ulcerative lesions in three patients with proved granuloma inguinale were healed by the use of this antibiotic. Finally, fifteen $Q$ fever patients were treated with oral aureomycin by a group of Califormian workers. In fourteen patients improvement occurred relatively promptly after commencement of the therapy, and the investigators conclude that oral aureomycin therapy offers considerable promise in the treatment of this fever.

Although it may be unwise to do so, one is tempted to try to assess the probable future importance of aureomycin in relation to three other antibiotics of proved clinical importance, namely, penicillin, streptomycin and chloromycetin from Streptomyces venezuelae. In the treatment of most Gram-positive bacterial infections, the supreme position of penicillin remains unchallenged. Streptomycin appears to hold a similar though less exalted position in the treatment of many tubercular infections; but it seems probable that aureomycin may displace streptomycin in the treatment of many Gram-negative infections, par. ticularly of the urinary tract, because of the unquestioned advantage it possesses that bacteria do not become resistant to it in vivo as they often do to streptomycin. In the treatment of viral and rickettsial diseases, both chloromycetin and aureomycin offer considerable grounds for hope in infections which have so far proved very resistant to chemotherapeutic agents. Whether these two antibiotics will prove to be competitive with, or supplementary to, each other only the future can tell. In the meantime, British investigators will wish to congratulate their American colleagues in the Lederle Laboratories on a very noteworthy achievement. HAROID RAISTRICK

\section{Hels \\ POST-GLACIAL CLIMATIC CHANGE}

A WELL-ATTENDED joint meeting of the Royal Meteorological of $Q$ Royal Astronomical Societies on December 1 pliftussed recent work on changes of climate singe th Ice Age. The discussion was opened by Mr. F 17 yle, who sid that any persistent change in the teperatur of the earth's surface must depend utimaloly on rabation from the sun. Without some extornal cause, solar radiation would not change appreciably in many millions of years, neither would the mean distance of the earth from the sun. But the sun travels through a gaseous medium which is aggregated locally into clouds, and in passing through such a cloud it would sweep up a number of particles. The kinetic energy of these, falling into the sun, is converted into heat, which might increase total solar radiation by as much as ten per cent. All this excess energy appears in the ultra-violet part of the spectrum, but as the latter normally forms only one tenthousandth part of the solar radiation, passage through a cloud would magnify the ultra-violet many times. This radiation is absorbed in the higher levels of the atmosphere and might alter its whole behaviour, causing great changes of climate.

The strongest evidence for post-glacial changes of climate is biological, and Prof. H. Godwin described the analysis of frequencies of pollen grains. These show the successive incoming of birch, pine and hazel, elm, oak, lime and alder, indicating a progressive amelioration of climate. The sudden expansion of alder and lime about 6000 B.c. marked the beginning of the Post-glacial Climatic Optimum, a warm period which continued until about 3000 B.c., after which the climate again deteriorated. There was a rather sudden worsening about 500 B.C., with flooding horizons and other evidence of increased rainfall. But the interpretation of biological evidence is sometimes difficult. It depends mostly on the present range of the species; but the regional range may be confused with what Prof. Godwin termed the 'microclimatic range', which allowed of local occurrences in exceptionally favoured spots outside the main range.

Prof. Godwin gave an interesting example of a new technique in the analysis of floras, in the climatic control of holly, ivy and mistletoe. A number of meteorological stations are selected and the performance of nearby plants noted-whether they bear ripe fruit regularly, occasionally or never, or whether the plant is not found in the district. These are plotted on diagrams against the mean temperatures of the warmest and coldest months. In this way the 'thermosphere' of each species is defined. Holly and ivy are controlled by the winter temperature, mistletoe by the summer temperature. Prof. Godwin illustrated a region in which the two former are at present flourishing, while mistletoe does not ripen its fruits (mild winters, cool summers). In the Sub-boreal, jvy and mistletoe flourished but not holly (cold winters, hot summers). During the Climatic Optimum, however, all three plants ripened their fruits, indicating mild winters and warm summers.

Climatic changes in the historic period were described by Prof. Gordon Manley, who compared long instrumental records of temperature with advances and retreats of glaciers. The greatest postglacial expansion of glaciers began in Scandinavia so late as 1750, in the Alps about 1550 and in Iceland probably as early as 1350. Glaciers are now every. where in rapid retreat, and in Norway archæological remains have been uncovered dating from 1300 . Long records of temperature have been carried well back into the eighteenth century at Stockholm, in Holland, and by Prof. Gordon Manley himself in Lancashire. There are difficulties of exposure and of local change of climate due to urbanization, but the general agreement is sufficient to show that the major oscillations are real. The principal feature has been a rise of winter temperature while the summer temperature has maintained a generally constant level; the annual range has everywhere decreased. The temperature records fit in very well with the records of glaciers; in particular, the chilly springs about 1740, which were general and in places catas. trophic, decreased the ablation period for glaciers and were followed by glacial advances.

Prof. Gordon Manley then discussed possible causes. Dr. Angstrom considered that periods of maritime climate were due to increases of the atmo. spheric circulation, but this only carries the problem back one stage. Moreover, the great fall of temperature about 500 B.c. requires some other factor, and this may be found in the spread of Arctic ice and melt-water. Low spring temperatures result from the frequent inflow of polar maritime air; mild winters with little frost are associated with maritime tropical air, and resemble the conditions in the 